\section{EFEITOS DE NIVEIS CRESCENTES DE CANA DE ACUCAR (SACCHARUM OFFICINARUM L.) NA DIETA DE OVINOS, SOBRE A DIGESTIBILIDADE DOS NUTRIENTES}

ROMUALDO SHIGUED FUKUSHIMA Professor Assistente Faculdade de Medicina Veterintria Zooteenia da USP

MARCUS ANTONIO ZANETTI Professor Assistente Doutor Faculdade de Medicina Veterinaria Zootecnia da USP

CARLOS DE SOUSR LUCE: Professor Ti tular Faculdade de Medicina Veterinaria Zootecria da USP

CATARINA ABDALLA

Auxiliar de Ensino

Faculdade de Medicina Veterinaria Zootecnia da USP

FUKUSHIMA, R.5.; ZANETTI, M.A.; LUCEI, C.S.; ABDaLLA, C. Efaitos oe niveis crescenter de cana de eccear (Sacetarum officinarue L.) ne dieta de ovinos, soore a digestibilidad dos nuerientes. Rev. Fac. Med. vet. Zootec. Univ. 5. Poulo, 23(2):i61-166, 1986

RESUMO: For an estudados as alteractes decoprentes do uso de cana de acuear en suostituiego silagem de milno, no consumo voluntario de materia seca enadigestibilidade uperente dos nutrientes, utilizando 9 carnejios, d stribuldos an 4 tratamentas: A) somente silagem: B) 668 de sitagem 335 de cana: C) 333 de silagam 66s de Eano: J) somento cana. Para tornop os dietas isooroteicas, foi utilizado farelo ol soja. Nyo nouve difserence significativa ouer no consume, quer na digestibilidade da mathria seca. A digestibilidade dos extrativos nzo nitrogenedos fol major no tratamento $D$ que no tratamento $A$. DE valopas medios dos conficientes de digestibllle oade para os trationentos A,B,C D, foram resoectivamente: mothis secs 49,31: 48,35, 54,78. 53,85 ; oroceina bruta 46,$04 ; 55,53 ; 56,24$ : 54,49 ; extrato elereo 50,89; 59,54; 55,77: 47.325: fiora jruta 49,42; 39, 39; 45,40,44,783; extrativos nao ndtrogenedos 54,58,55,73, 60,30. 64,008 .

UNITERMOS: 9 . Nutrleso, ovinos; 2 . alimentos, canade actear:T3. Olgestibilidede

\section{INTRODUEAO}

A earactertstica de manter a digestibilidade alta com o avanco oa maturioade da obanta, confere uma imoortante vantagem para a cana de acucap (Sacenarum ofilicinapum L.) como alimer.to destinado a ruminantes (PRTE, 15 e PRESTON, 20), particularmente na estacalo das secas, quando outras grantneas dectinam tanto em quantidade como - qualidade (PEDREIRR, 17). Na verdade, a pratica de usar eana picada ou desintegrada, na perloda da estiagem, e comum entre os criadores, sendo fornecida isolada, misturada com silagem de milno, ete. Devese levar en conta, a alta produca de materia seca, freaóntemente excedendo 40 ton/ha (PRESTON et alii, 19).

Segundo GOODING, 7, adesar de varios estudos apresentarem a cana como tendo cerca de 608 de nutrientes digestiveis totais (NDT) na materia seca, os resultacos experimentais nao szo comaltveis com tais cifras. Eana, en suostituigzo parciab ou total a outros volumosos, tem se mosisado menos f avortuel no desemoenho animal; $j$ a em 1940, ATHANR550F, 3 , demanstrou es te $f$ a to para vacas em lactacgo, quando confrontou a cane con a mandoca con caoim fino. Mais recentemente, para gado de corte, VELLDSO, 21, verificou que a cana, como volumoso exclusivo, foj o que apresentou pior desempenho, quando comparada com silagens de milho ou de sorgo e pe de milho seco.

BIDNDI $t$ ali $i, 4$ NAUFEL it ali $i, 13$ observaran que vacas en lactacyo consumiram menas materia seca, quando o volumoso foi a cana, en relasto a silagen de milho. Este aspecto tamben foi constatado oor NOGUEIRA FILHO et alij, 14, quando sudstituiram a cana pela silagem de sorgo. R medida aue majores prooorcoes de cana eritraram nas dietas, PRTE, 15, tamoem verificou menor consumo de materia seca por novilhos.

Entretanto, gOIN at alii, $5, \quad$ HOCHSTRASSER et alij, 9 , ooservaram que bovinos leiteiros consumiran mais materia seca na forma de cana, do que na de silagen de milho. De maneira identica, earneiros lngeriram maiores quantidades de cana, a medida que esta suostituiu pontas de cana (YOUSSEF \& RASTOGI, 22). ARANIA, 1, verificou que o consumo de materia seca da cana foi melhorado pelo uso de concentrado (farelo de arroz).

PEDREIRA, 15, obteve os seguintes coefleientes de digestibilidade cem carneipos), da cana forrageira IAC 3625: materia seca 51,968; extrato etereo 36,538; fibra bruta 35,858; extrativos nao nitrogenados 64,108 a protelna bruta 5.518. MORRISON, 11. eitjos seguintes coeficientes de digestibilidada para a cana: protelna bruta 578; extrato etereo 598; ribra bruta 518 . extrativos nzo nitrogenados 638.

0 efeito do emorego de pontas de eana en substituiczo a cana, para carneiros, foi de diminuir a digestibilidace oe materia 
seca, eujo valor para o tratamento contendo somente cana como volumoso, foi de 63,78 (YOUSSEF \& RRSTOGI, 22).

BOIN et alij, 5, verjficaram nao exisia diferencas na cigestibilioade ca materia seca e proteina bruta, em novilnos mesticos recedendo sibagem de nilno ou eana; no entanto, o tioo de volumoso influiu significativamente os coeficientes da fibra bruta $(56,0 \times 41,78)$, extrato etereo $(82,7 \times 74,98)$ e extrativos nao nitrogenados $(68,6 \times 74,45)$, respectivamente papa silagen e cana.

MELDTTI, 10, em un estudo comparativo da dioestibilidade da cana forpageira IAC 3625, obteve os seguintes coeficientes para ovinos e bovinos, respectivamente: materia seca 61,83 e 62,78\%; protelna bruta 12,41 e 8,798; extrato etereo 83,12 e 86,258 ; fibra bruta $47,22 \%$ e $40,22 \%$ extrativos nto nitrogenados 72,89e 75,34\%. Rpenas o componente extrato etereo opresentou diferenca estat:5:ica a favor dos bovinos.

D presence traoalno objetivou medir as alteractes decorrentes do uso da cana de afucar, en substituislo a silagem de milho, no consumo de materia seca, bem como na digestibitidade aperente dos nutrientes. Estes aspectos merecen maiores estudos, notadanente. em palses de elima tropical, onde a cana produzida, poaende servir como importante recurso alimentar para ruminantes.
MATERIAIS E METODO

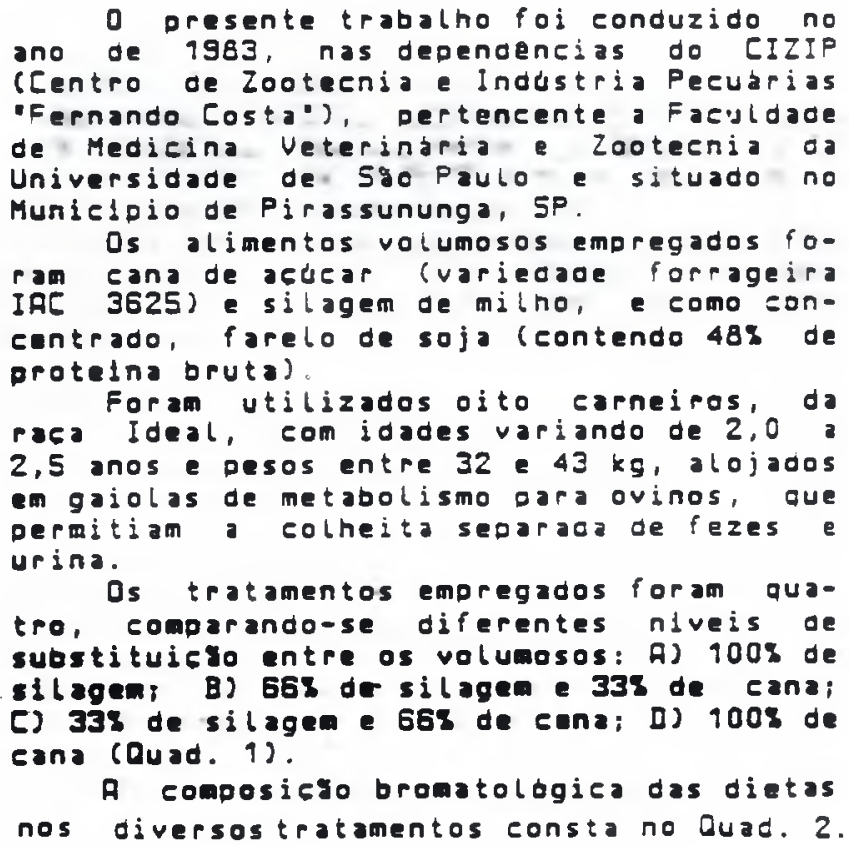

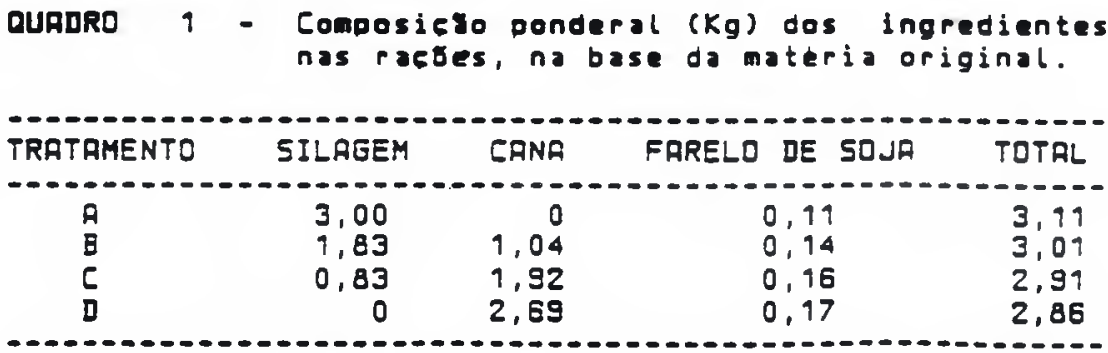

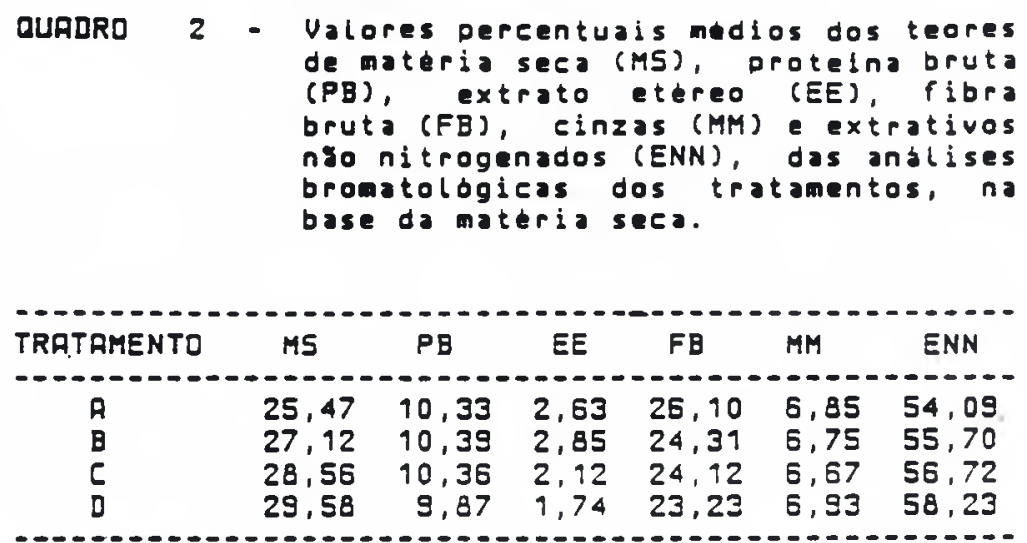


Os volumosos eram auito bem misturados, segundo os niveis de substituigro, a eles incorporados, além do farelo de soja, cerca de cez gramas de una mistura em oartes aguais, de 5 al comum e sudemento vitaminis=o-minerab isomposisao por $\mathrm{Kg}=$ saicio 240.0 g; fosforo 180,0g; magnerio 12,0g; sodio $12,5 \mathrm{~g}$ i ferro $5500 \mathrm{mg}$ i zinco $2400 \mathrm{mg}$; manganes $2200 \mathrm{mg}$; cobre $500 \mathrm{mg}$; cobalto $300 \mathrm{mg}$; iodo $200 \mathrm{mg}$; fluor $1800 \mathrm{mg}$ e vitamina $A 500.000$ U.I.J.

Us abimentos foran fornecidos, de acordo com as necessidades de mantenca dos animais, obedecendo-se as normas do NATIONAL RESERRCH COUNCIL, 12.

Para o ealeulo da digestibilidade dos nutrientes, foi utilizado o metodo da colheita total de fezes. As fezes foram colhidas nos oltimos cinco dias de cada subperiodo experimental e as amostras de alimentos e sabras, nos altimos sete dias. Das fezes coletadas diariamente, foram amostradas 208 censervadas en freezer ate as andises. As analises bromatolbgicas foran canduzidas de acordo com as normas oa A5SDCIATION OF OFFICIAL ANALYTICAL EHEMISTS, 2, e os ENN ealeulados por diferenca. O consumo de alimentas foi anotado diariamente, no decarrer de cada sub-perfodo experimental. o delineamento experimental usado foi um quadrado latino $4 \times 4$, conforme PIMENTEL GOMES, 18, com 2 grupos de 4 animais cada. Foran desenvolvidos 4 sub-perlodos com duracro de 25 dias eada, perf́azenco um total de 140 dias. As analises ce variancia foram de acordo com a metodologia deserita por HARVEY, 8. Na interpretaczo estatistica, as diferencas entre medias foram detectadas pelo teste de Tukey, ficando estadelecido, "a priori", que a significancia estatistica seria considerada ao nivel de 58 de probabilidade ( $p \leq 0,05)$.

\section{RESULTADOS E DISCUSSAD}

D consumo de alimentos, exoresso em gramas de materia seca ingeridas dor animal por dia (MS/an/d) e gramas de materia seca ingeridas por quilo de peso metabobiso (MS/kg 0,75), dentro dos tratamentos, estzo ne Tab. 1 .

$\begin{aligned} \text { TAEELA } 1 \text { - } & \text { Consumo medio de materia } \\ & \text { seca por animal por dia } \\ & \text { (MSIan/d) de materia seca } \\ & \text { por quilo de peso, vivo } \\ & \text { metabolico (MS/kg ). }\end{aligned}$

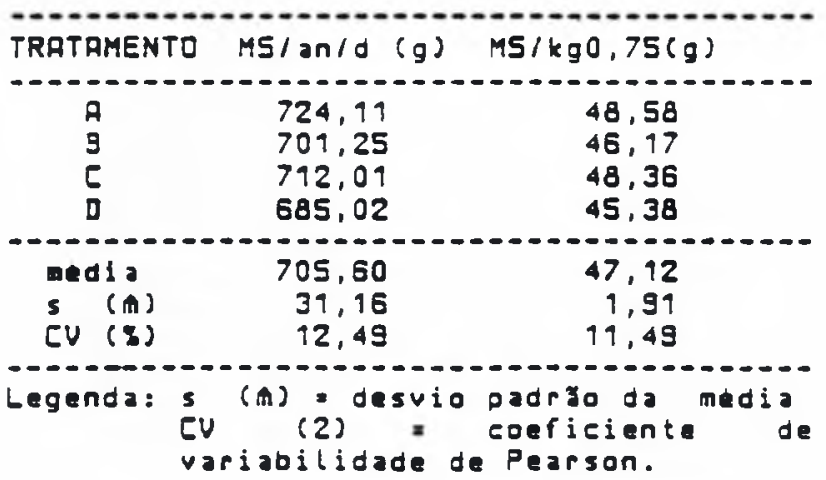

Nao houve diferencas ( $0 \leqslant 0,05)$ no consuma de materia seca que pudessem ser devidas aos tratamentos. Deonsumo medio de materia seca no tratamento d foíd de 385,02 glan/d, ligeiramente inferiar do obtido por ARANDR, $1(780 \mathrm{~g})$; do mesmo modo, o consumo de MS/kg 0,75, que atingiu 45,38 g, foi inferior aos valores de 62 a $69 \mathrm{~g}$, abtidos por YOUSSEF \& RRSTOGI, 22, com dietas onde as pontas de cana substitulpam pareial e totalmente a cana.

Rpesar de náo terem sido cetectadas diferencas significativas, a meaida que o nivel de cana aumentou na dieta, en substituifso silagen de milho, hauve tendencia de aueda no consumo de materia seca. Resultados semelhantes foram obtidos por BIONDI et alii, 4, NAUFEL et alii, 13 e NDEUEIRA 
164 FUXUSHIMA. R.S.: ZANETTI. M.A.; LUCCI. C.S.; ABDALIA, C.

FILHD et alij, 14, trabalhando com bovinos. D menor consumo, relatado com a utilizaczo de cana, poderia ser explicado pela baixa degradasyo ruminal de sua fibra (PRTE, 15). No entan:c, EJIN e: ali:, 8 e HEEHSTRRS5ER et abij, 9, encontraram resuitaoos dfferentes, com os bovinos ingerindo maior quantidace de oe materia seca na forma de cana, do que na de silagem de milho.

No que diz respeito a digestibilidade aparente dos nutrientes, o coeficiente de digestibilidade a materia seca foi bem uniforme entre os tratamentos (Tab. 2). adresentanoo valor medio de 51,69\%; iguatmente, $30 I N$ et alid, 5, verificaram no existir diferencas nos lndices de materia seca digestivel entre cana e silagem de milho, para novilhos mesticos. D valor obtido para o tratamento contendo cana como onico volumoso, foj de 53,858, semelhante ao valor reportado Dor PEDREIRA, 16 (51,968), mas inferior 305 de Youssef \& RRSTDEI, 22 (E3,78) e MELDTTI, 10 (61,83\%).

o coeficiente de digestibilidade da protelna bruta (Tao. 2) foi joual entre os tratamentos, excecto fejta ao tratamento $C$, estatlsticamenee superior $(p<0,05)$ ao $A$, sem motivo aparente. BDIN et alii, 5 nso encontraran diferencas na digestibilidade da protetna bruta, en novilhos mesticos recebendo dietas de cana ou silagen de milho. 0 coeficiente pera o tratamento contendo so eana como volumoso (54,49:) foj semelhante aos dados fornecidos por MORRISON, 11 (57\%), Dorem, maior ao relatado Dor MELOTT!, $10(12,49$ A) e DOP DEDREIRF, 1 S (5,618), Dossivelmente pelo fato ae no presente experimento ter se lancado mao de um concentrado proteico, o farelo de soja. que fol oferecido misturado a volumoso, una vez que a proteina da cana de baixa dlgestibilidade.

NFo houve diferencas no coeficiente de digestibilidade da fracao extrato eiereo entre os tratamentos, cujo valor medio foj de 53,59\%. O valor obtido no tratamento D (47,B28) foi proximo ao que consta em MOFRISON, Iq (51\%), mas superior ao obtido por PEDREIRA, $16(36,83 \%)$ e inferior so do MELDTTI, $10(83,128)$

A digestibilidade da fibra bruta foi estatisticamente diferente $(p \leq 0,05$ ) entre os tratamentos (Ouad, 4). Embora nyo esti. vesse bem caracterizaoa, houve tendencia dos tratamentas contenoo cana, aresentaren menor digestibilioaoe da fiora bruta, como j) foi assinalado por PATE, 15. O coefi. clente do tratamento cantendo so cana como volumoso foi de $44,78 \%$. maior que o obtido por PEDREIRA, $16(35,85 \%)$, mas menor que o dado fornecjdo por MORRISON, 11 (518); sendo, no entanto, concordante ao de MELDTTI, $10(47,65 \%)$.

TABELA 2 - Valares medios dos coeficientes de digestibilidade aparente $(x)$ da nateria seca (MS), proteina bruta (PB), extrato etereo (EE), Pibra bruta (FB) e extrativos ngo nitrogenados (ENN), na base de materia seca.

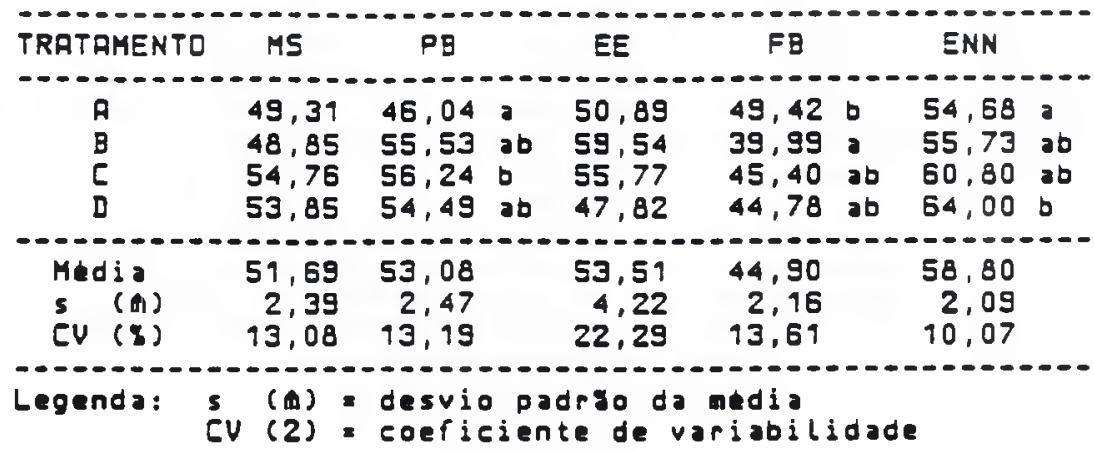

Medias seguidas por letras diferentes, para uma mesma caracteristica, diferem entre $s 1(p<0,05)$, pelo teste de Tukey.

Houve tendencia de melhor digestyo dos extrativos nao nitrogenados, nos tratamentos contendo maiores prodorezes de cana, pois esta aumentou os teares de carboidra- tos soluveis oestes iratamen:os; aestar:e, a digestibilidade desta fracgo, no tratamento contendo somente cana, foi significativamente major $(p \leqslant 0,05)$ en relafyo ao contendo somente silagem de milho como volumoso. O coeficiente de digestibilidade, obtido no oresente experimento para tratamento $D$, foi de 54,008, concordando 
com os valores citados por MORRISON, 11 (63\%) Dor PEDREIRA, $16(64,10 \%)$; entretanto, foi inferior ao descrito por MELDTTI, $10(72,89 x)$.

\section{EONCLUSOES}

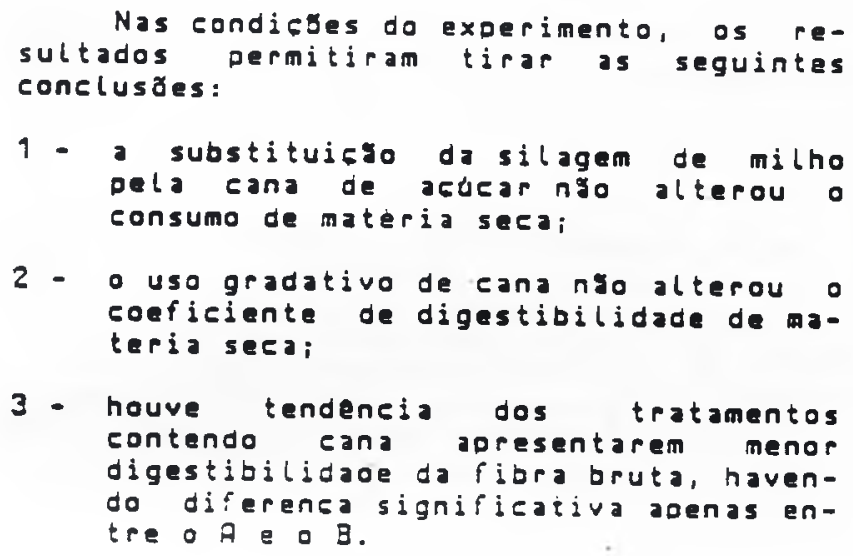

3 - houve tendencia dos tratamentos contendo cana apresentarem menor digestibilidaoe da fibra bruta, havendo diferenca significativa adenas entre o a o o $B$.

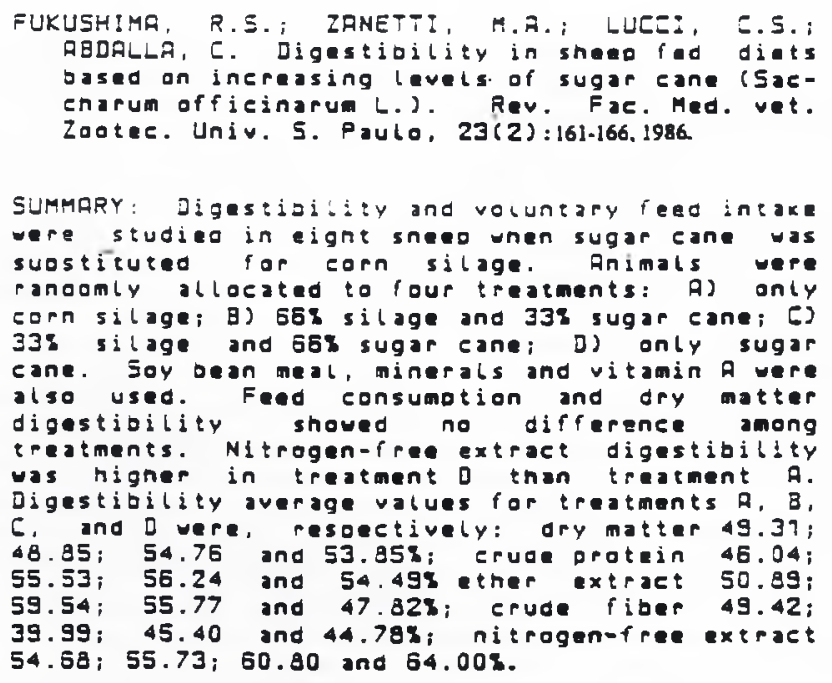

UN!TERMS: 9 . Nutrition of sheeo; 2 . Food, sugar eane: ${ }^{+}$. Podarent digestiolitity

REFERENCIRS BIBLIDGRAFICAS

1 - ARANIR, E. Voluntary consumation pattern and its relation with pumen fermentation in sheep fed diets based on sugar cane. Trop. ania. Prod., 2:232. 1977. CResumo)

2 - ASSDCIATIDN OF OFFICIAL ANALYTIEAL CHEMISTS. Offielal methads of analysis. 12.ed. Washington. 1975 .

3 - ATHANAS5DF, N. A cana na alimentaço dos animais domesticos. Rey. Agrte., Syo Paulo, 15:421-427, 1940 .

4 - GIONDI, P.; EAIELLI, E.А.; FREITAS, E.A.N.; LUCEI, C.S.; ROCHR, G.L. Substituiczo pareial e total da silagen de milho por cana de acdcar como unicos volumosos para vacas em lactacro. B. Industr. anim., 35:45-55, 1978 .
5 - BOIN, E.; ALLEONI, G.F.; BEISMAN, D.R. Comoaraço entra silagem de nilho e cana de acdear na alimentacso de ruminantes. 1. Digestibilidade de racóas balanceadas. In: REUNIAO ANUAL DA SOCIEDADE BRASILEIRA DE ZOOTECNIA, 20.. PElotas, 1983. Anais. p.83.

6 - BDIN, C.; ALLEDNI, G.F.; BIONDI, P.; BÚNILHA NETO, L.M. Comoaracgo entre silagem de milho e cana de actear na alimentacazo de ruminantes. 2. Ef eito do nlvel de concentrado na producga de leite. In: REUNIAO RNUAL DA SOCIEDADE BRASILEIRA DE ZOOTECNIA, 20., PElotas, 1983. Anats. p.84.

7 - GOODING, E.G.3. Efecto de la calidad de la caña soore su valor somo alimento para jovinos. Prod. anim. Trop., 7:76-97, 1982.

8 - HARUEY, W.R. Last-squares ari-bysis of data with unequal subelass

Rev.Fac.Med.vet.Zoorec.Univ.S.Paulo, 23(2):161-166, 1986. 
166 FUKUSHIMA. R.S.; ZANETTI. M.A.; LUCCI, C.S.: ABDALLA, C.

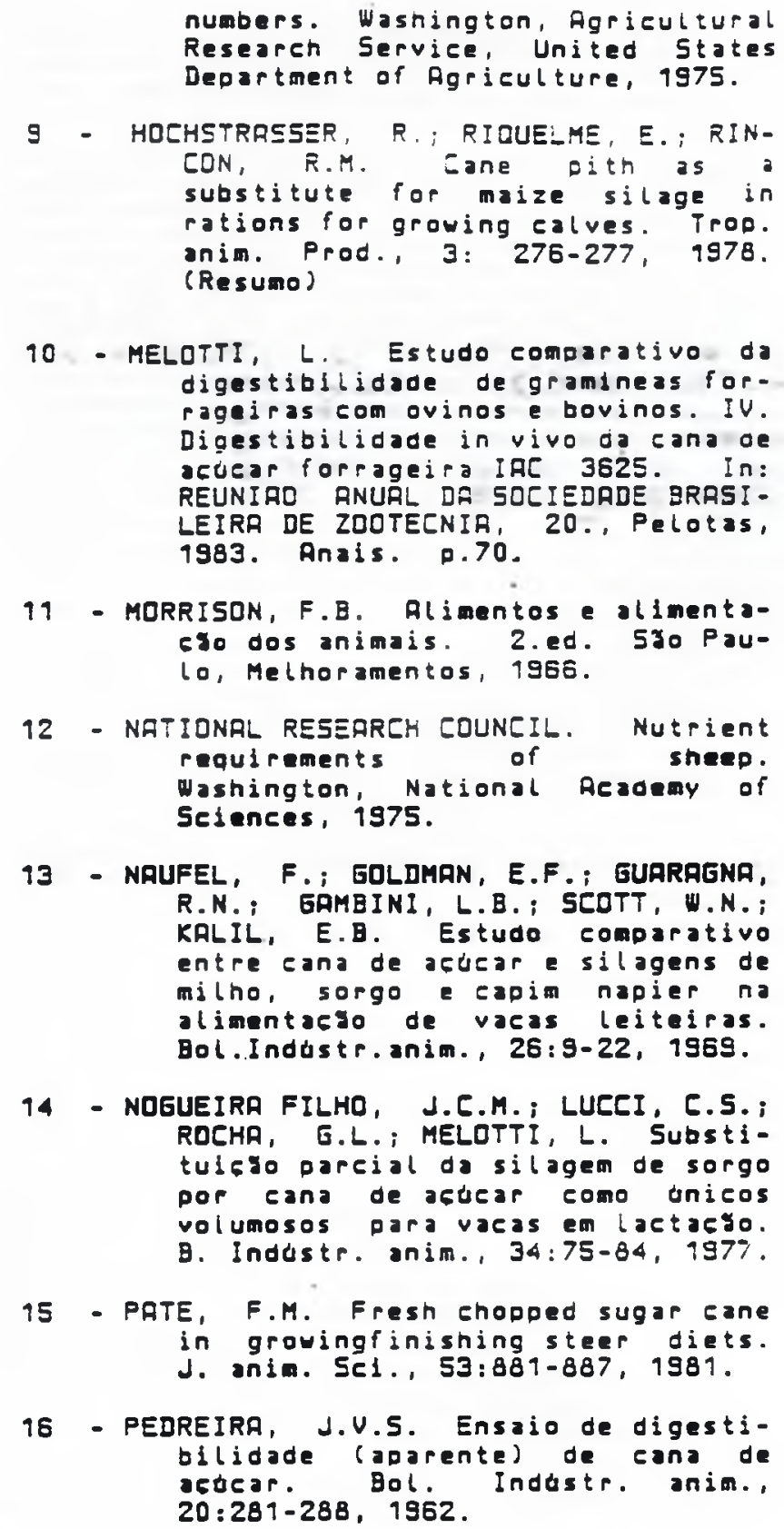
REUNIRO RNURL DA SOCIEDRDE QRRSILEIRA DE ZDOTEENIA, 20., Pelotas, 1983. Anais. D.70.

11 - MORRISON, F.E. Alimentos e alimentacso dos animais. 2.ed. Sro PauLo, Methoramentos, 1956.

12 - NATIONRL RESERREH COUNEIL. Nutrient requirements of sheep. Washington, National Reademy of Sedences, 1975 .

13 - NAUFEL, F.; GOLDMAN, E.F.; GUARAGNA, R.N.; GAMBINI, L.B.; SCOTT, W.N.; KALIL, E.B. Estudo comparativo entre cana de acucar e silagens de milho, sorgo e capim napier na alimentacio de vacas leiteiras. Bol.Industr. anim., 28:5-22, 1569 .

14 - NDEUEIRA FILHO, J.C.M.; LUCEI, C.S.; ROCHA, G.L.; MELDTTI, L. Substituifjo parcial da silagem de sorgo por cana de acucar como unicos volumosos para vacas em lactacyo. 8. Industr. anim., 34:75-64, 197\%.

15 - PRTE, F.M. Fresh chopded sugar cane in grouingfinishing steer diets. J. unia. Sc1., 53:881-887, 1981 .

16 - PEDREIRA, J.V.S. Ensaio de digestibilidade (aparente) de cana de actear. Bol. Indastr. anian., $20: 281-288,1962$.

17 - PEDREIRA, J.V.S. Crescimento estacional dos eapins coloniyo Panicum andimum Jaca., goroura Melinis minutiflora Pal. beauv., jaragua Hyparrhenia rufa (Ness) 5 tadf.e pangola de taiwan fi-24 Digitaria pentzil 5 tent. Bol. Industr. anim., 30:59-945, 1973.

18 - PIMENTEL GOMEs, F. Curso de estatistlea experimental. G.ed. 5yo Paulo, Nobel, 1981.

$1 S$ - PRISTON, T.R.; CARCANO, C.; ALUAREZ, F.J.; GUTIERREZ, D.G. RIEE polishings as a supplement in a sugar cane diet: effect of level of rice polishings and of processing the sugar cane by derinding or ehopping. Trop. anim. Prod., 1:150-162, 1976.

20 - PRESTON, T.R. Nutpitive value of sugar cane for ruminants. Trop. anim. Prod., 2: 125-142, 1977.

29 - VELLO50, L. Estudo comparativa sobre - valor das silagens de milho e sorgo, do "pl de mitho" e da cana desintegrada fornecidos a novilnos Nelore em confinamento. Bol. Industr. anim... $27 / 28: 313-313$, $1970 / 1971$.

22 - YOUSSEF, F.E. \& RRSTOGI, R.K. Voluntary intake and digestibility in sheep given rations containing chopped or derinded sugar cane stalks and cane tops in different proportions. Trop. anim. Prod. 3:273, 1978. (Resumo)
Recebido para publicacáo em $10 / 7 / 85$ Aprovado para publicacyo em $27 / 8 / 86$ Impresso en $12 / 86$ 